\title{
FORMULATION AND EVALUATION OF HERBAL ORAL EMULGEL CONTAINING PSIDIUM GUAJAVA LINN. LEAVES EXTRACT (A PREVENTIVE ORAL CARE PREPARATION)
}

\author{
AKBAL AHMAD*, ABADHESH KUMAR NIRANJAN \\ Department of Pharmaceutics, Hygia Institute of Pharmaceutical Education and Research, Lucknow, Uttar Pradesh, India. \\ Email: akbalahmad480@gmail.com
}

Received: 23 September 2021, Revised and Accepted: 01 November 2021

\section{ABSTRACT}

Objective: The present research work is based to establish the formulation and evaluation of herbal oral emulgel containing extracts of powdered Psidium guajava Linn. leaves extract.

Methods: The guava leaves were collected and extracted by cold maceration process using different solvents, further ethanolic extract was used for the emulgel preparation. Emulsion and gel phase were prepared separately and mixed together in ratio of 1:1.

Results: Different formulations ere developed and evaluated for the physical appearance, pH, homogeneity, spreadability, viscosity, extrudability, antibacterial activity, and in vitro skin permeability studies. In all formulated emulgel containing P. guajava Linn. Leaves, extract F2 formulation gives all satisfactory results as in discuss in this paper.

Conclusion: Overall, concluded that the extract might contain anti-bacterial properties and further tests are required to prove for oral care activity using other in vivo or in vitro techniques.

Keywords: Emulgel, Carbopol, Antimicrobial, Franz diffusion cell, Permeability.

(C) 2021 The Authors. Published by Innovare Academic Sciences Pvt Ltd. This is an open access article under the CC BY license (http://creativecommons.org/ licenses/by/4.0/) DOI: http://dx.doi.org/10.22159/ajpcr.2021v14i12.43218. Journal homepage: https://innovareacademics.in/journals/index.php/ajpcr

\section{INTRODUCTION}

From the past several decades, the treatment of illness has been managed by administration of drugs to human body through various routes, namely, oral, sublingual, rectal, parental, vaginal, etc. The topical route of drug administration system is usually used where these systems of drug administration not functioning or in local skin infection such as fungal and other skin infection $[1,2]$.

Topical drug delivery can be defined as the application of a drug containing formulation to the skin to directly treat cutaneous disorder. From the major groups of semisolid preparations, the utilization of transparent gels has expanded both in cosmetics and in pharmaceutical preparations.

Gels are a remarkably newer class of dosage form created by entrapment of enormous amounts of aqueous or hydroalcoholic liquid during a network of colloidal solid particles.

Gel formulations generally provide faster drug release in comparison with other semisolid preparations such as ointments and creams [3].

In spite of the many advantages of gels, a serious limitation is within the delivery of hydrophobic drugs. Hence, to beat this limitation, emulgels are prepared and with their use even a hydrophobic drug can enjoy the unique properties of gels. When gels and emulsions are used in combined form, the dosage forms are referred as emulgel [4].

\section{METHODS}

The leaves of the plant Psidium guajava were collected from the local area of the horticulture department of Aliganj, Lucknow, Uttar Pradesh, India, in January 2021, and the plant specimen is authenticated by "CSIR-National Botanical Research Institute, Lucknow" Accession No. LWG 108272. All other ingredients of analytical grade procured from Hygia Institute of Pharmaceutical Education and Research laboratory.
Preparation of plant extract

The freshly collected guava leaves were washed for the removal of dirt under running tap water after that kept for shade drying for 3-4 weeks at room temperature. After drying with a mechanical grinder, the dried guava leaves were reduced to a coarse powder and passed by a sieve no. 40. Each $100 \mathrm{~g}$ of dried guava leaf's powder was macerated in $250 \mathrm{ml}$ of each ethanol, chloroform, and water in a conical flask at room temperature for $24 \mathrm{~h}$ at occasionally shaking. After $24 \mathrm{~h}$, mixture was filtered out using a simple filtration method and filtrates were collected in separate vessels. To obtain the extract, the solvent was removed from the filtrate under compact pressure using a rotatory vacuum evaporator at $45-50^{\circ} \mathrm{C}$

\section{Preparation of herbal emulgel}

Preparation of emulsion phase

The oil phase of the emulsion was prepared by dissolving Span 80 in glycerine and aqueous phase was prepared by dissolving Tween 80 in purified water [4]. Clove oil was added into oil phase [5]. Both the oily and aqueous phases were separately heated up to $40-50^{\circ} \mathrm{C}$, then the oily phase was mixed with the aqueous phase with continuous stirring until cooled at room temperature.

Preparation of gel phase

In a beaker, a specified quantity of Carbopol 934 was taken into $25 \mathrm{ml}$ of purified water and kept the beaker at bay for $1 \frac{1}{2} \mathrm{~h}$ to stir the Carbopol. In another beaker, $5 \mathrm{ml}$ of purified water was taken and heated with water bath by adding the required quantity of methylparaben and propylparaben. The solution was cool, and then, propylene glycol 400 was added. An additional amount of extract required was added to the above mixture and mixed correctly with a continuous stirring. Finally, a volume of up to $50 \mathrm{ml}$ was added to formulas for adjustment of the required mouth $\mathrm{pH}(6.8-7.3)$ to obtain the required consistency of gel by adding remaining distilled water and triethanolamine. 
Mixing of emulsion and gel phase

To obtain an emulgel, combine the gel with emulsion in a ratio of 1:1.

\section{Formulation table emulgel}

\section{Characterization of emulgel formulation}

The physicochemical and safety evaluations of all the formulations of $P$. guajava were carried out as follows.

\section{Physical characterization of emulgels}

The emulgels were smeared on glass slides as a thin layer and examined based on visual inspection. The color, phase separation, and grittiness of all the formulations were investigated [6].

\section{Measurement of $\mathrm{pH}$}

By adding triethanolamine at room temperature, the $\mathrm{pH}$ of all emulgel formulations was modified to neutral $\mathrm{pH}$. For determining the $\mathrm{pH}$ of emulgel formulations, a digital $\mathrm{pH}$ meter was used. Each formulation was determined at room temperature $(1 \mathrm{~g}$ of emulgel dispersed in $100 \mathrm{ml}$ of distilled water) [6].

\section{Viscosity}

The viscosity of all the jellified emulsion formulations was evaluated using spindle type Brookfield Viscometer (Model LVDV-E), at $25^{\circ} \mathrm{C}$ at an rpm of 10.

\section{Spreadability}

Spreadability represents the magnitude of the space where gel easily scatters when applied to the epidermis. The determination of spreadability is important in assessing the gel characteristics as it influences the remedial competence of the emulgel formulation [7]. It is indicated by the time consumed by two slides to get rid of the emulgel which was smeared along the space separating the slides, under the direction of a fixed weight. Shorter the time required for detachment of the two slides, the well the spreadability. The spreadability apparatus used was as suggested by Mutimer et al. (1956). Two glass slides of uniform dimensions were taken. One glass is fixed on the ground. About $2 \mathrm{~g}$ of the emulgel formulation was placed over the ground slide. The second glass slide was then placed over the ground slide in such a way that the emulgel was sandwiched between the two slides. A 1 $\mathrm{kg}$ mass was then placed on the two slides for 5 min. This resulted in the formation of a uniform film of emulgel without any entrapped air The surplus emulgel was discarded. The glass slide on the top was then tethered with a weight of $80 \mathrm{~g}$. Recorded the time (in seconds) taken by the top slide to move a distance of $7.5 \mathrm{~cm}$. Spreadability of the emulgel formulations was estimated:

$S=M \times L / T$

Where, $\mathrm{S}$ - spreadability (gram.cm/sec.), $\mathrm{M}$ - weight fixed to top slide ( $80 \mathrm{~g}$ ), $\mathrm{L}$ - distance progressed by the glass slide $(\mathrm{cm})$, and $\mathrm{T}$ - time (in seconds) taken by the top slide to move a distance of $7.5 \mathrm{~cm}$.

\section{Extrudability}

The formulated emulgels were filled in collapsible tubes. Extrudability of the emulgel formulations was performed to determine the force required to expel the contents of the tube. It indicates the ease with which the emulgel can be ejected from its container. It is expressed as the weight needed to squeeze out a narrow strip of emulgel $(0.5 \mathrm{~cm})$ within $10 \mathrm{~s}[7,8]$. The extrudability of all the formulations was measured thrice and the normal values were determined using the following formula;

$$
\text { Extrudability }=\frac{\text { Weight applied to extrudegel }(\mathrm{g})}{\text { Area of applied force }\left(\mathrm{cm}^{2}\right)}
$$

\section{Antibacterial activity}

The antimicrobial activity of emulgels of $P$. guajava was investigated using the technique of agar well diffusion $[9,10]$. Mueller-Hinton agar
(Sigma-Aldrich) was used as the medium. The microorganisms used for the activity of antimicrobials studies were Gram-positive bacterium (Staphylococcus aureus ATCC25923) and a Gram-negative bacterium (Pseudomonas aeruginosa ATCC 27853). The sterile agar plates were inoculated with the bacteria culture ( $S$. aureus and $P$. aeruginosa) under aseptic conditions at $37^{\circ} \mathrm{C}$, for $48 \mathrm{~h}$. A $6 \mathrm{~mm}$ diameter well was bored with the help of a sterilizer and individually filled with $100 \mu \mathrm{L}$ test formulations produced by dissolving dimethyl sulfoxide $(1000 \mu \mathrm{g} / \mathrm{ml})$ and the solvent control system (DMSO). The plates were retained before inoculation and incubated at $37^{\circ} \mathrm{C}$ for $24 \mathrm{~h}$ to allow the extracts to be pre-dispersed into the agar. After the incubation period, the diameter of the growth inhibition areas was measured. In comparison to a commercial product, the antibacterial activities of the formulations were (comparison with marketed antifungal formulation (Daktarin oral gel).

\section{RESULTS AND DISCUSSION}

\section{Physical characterization of emulgels}

The physical characteristics of gellified emulsions of $P$ guajava Linn. were examined visually. $P$. guajava Linn. emulgel was light yellowish-white in color. All the formulations appeared homogenous without any separation of both aqueous and oily phases. The formulated emulgels had a good aesthetic appearance, thus passing the pharmaceutical quality grade acceptance $[6,8]$.

\section{pH of emulgel formulations}

The $\mathrm{pH}$ of all of the emulgel formulations was found to be within 6.8-7.0. Those that did not meet the specified $\mathrm{pH}$ range were adjusted to the desired range using triethanolamine. Final $\mathrm{pH}$ value(s) of the emulgel formulations are presented in Table 2.

\section{Viscosity of emulgel formulations}

At room temperature, the viscosity of emulgel formulations was measured. P. guajava Linn. Carbopol-based formulations have shown higher viscosity. With an increase in gelling polymer concentration used, the viscosity of the emulgel formulations increases. The gelling polymers reduce interfacial and surface tension and thereby increase the viscosity of the liquid phase [11]. Viscosity values of P. guajava emulgels range from 3190 to 9772 cps. Viscosity values of emulgels are displayed in Table 3.

Table 1: Composition of various emulgel formulations containing extract of Psidium guajava Linn. leaves with Eugenia caryophyllus oil

\begin{tabular}{|c|c|c|c|c|c|}
\hline \multirow[t]{3}{*}{ Ingredients } & \multicolumn{5}{|c|}{ Quantity in gm } \\
\hline & $\mathbf{F 1}$ & F2 & F3 & F4 & F5 \\
\hline & g & g & g & g & g \\
\hline Clove oil & 1 & 1 & 1 & 1 & 1 \\
\hline Guava leaf extract & 1 & 1 & 1 & 1 & 1 \\
\hline Carbopol 934 & 0.5 & 1 & 1.5 & 2 & 2.5 \\
\hline Span 80 & 1 & 1 & 1 & 1 & 1 \\
\hline Tween 80 & 1 & 1 & 1 & 1 & 1 \\
\hline Methyl paraben & 0.06 & 0.06 & 0.06 & 0.06 & 0.06 \\
\hline Propyl paraben & 0.03 & 0.03 & 0.03 & 0.03 & 0.03 \\
\hline Propylene glycol & 5 & 5 & 5 & 5 & 5 \\
\hline Glycerine & 7.5 & 6.5 & 6.5 & 6.5 & 6.5 \\
\hline Triethanolamine & 0.1 & 0.1 & 0.1 & 0.1 & 0.1 \\
\hline Purified water & q.s & q.s & q.s & q.s & q.s \\
\hline
\end{tabular}

Table 2: pH of formulation

\begin{tabular}{ll}
\hline Formulation & pH of emulgel \\
\hline F1 & $6.92 \pm 0.10$ \\
F2 & $7.00 \pm 0.09$ \\
F3 & $6.90 \pm 0.11$ \\
F4 & $6.88 \pm 0.10$ \\
F5 & $6.88 \pm 0.08$ \\
\hline
\end{tabular}


Table 3: Viscosity of emulgel

\begin{tabular}{ll}
\hline Formulation & Viscosity of emulgel (cps) \\
\hline F1 & $3190 \pm 15.28$ \\
F2 & $3593 \pm 10.26$ \\
F3 & $3990 \pm 19.52$ \\
F4 & $8381 \pm 19.31$ \\
F5 & $9772 \pm 11.85$ \\
\hline
\end{tabular}

Table 4: Spreadability of emulgel

\begin{tabular}{ll}
\hline Formulation & Spreadability of emulgel (g.cm/s) \\
\hline F1 & $13.47 \pm 0.50$ \\
F2 & $13.83 \pm 0.06$ \\
F3 & $14.87 \pm 0.23$ \\
F4 & $23.17 \pm 1.04$ \\
F5 & $26.67 \pm 1.15$ \\
\hline
\end{tabular}

Table 5: Extrudability

\begin{tabular}{ll}
\hline Formulation & Extrudability $\left(\mathrm{g} / \mathbf{c m}^{2}\right)$ \\
\hline F1 & $11.13 \pm 0.90$ \\
F2 & $12.11 \pm 0.90$ \\
F3 & $12.43 \pm 0.55$ \\
F4 & $13.93 \pm 0.58$ \\
F5 & $14.30 \pm 0.85$ \\
\hline
\end{tabular}

Table 6: Antibacterial activity of the P. guajava Linn. emulgel formulations

\begin{tabular}{lll}
\hline Formulations & Inhibition zone in mm & \\
\cline { 2 - 3 } & Staphylococcus aureus & $\begin{array}{l}\text { Pseudomonas } \\
\text { aeruginosa }\end{array}$ \\
\hline F1 & $24.57 \pm 0.59$ & $24.83 \pm 0.25$ \\
F2 & $21.50 \pm 0.46$ & $21.50 \pm 0.46$ \\
F3 & $19.83 \pm 0.21$ & $20.0 \pm 0.44$ \\
F4 & $19.17 \pm 0.38$ & $18.77 \pm 0.32$ \\
F5 & $19.53 \pm 0.45$ & $19.20 \pm 0.17$ \\
Standard & $21.17 \pm 1.07$ & $21.40 \pm 0.89$ \\
drug (Daktarin oral gel) & & \\
\hline
\end{tabular}

Spreadability of emulgel formulations

Spreadability represents the magnitude of the space where gel easily scatters when applied to the site of application. The remedial competence of the emulgel formulation also relies on its spreadability. All the formulations exhibited reasonably good spreadability. Spreadability of values of P. guajava emulgels extended from 13.47 to $26.67 \mathrm{~g} . \mathrm{cm} / \mathrm{s}$ [12].

\section{Extrudability}

Extrudability indicates the ease with which the emulgel can be ejected from its container. It is the power required to push or force the contents out of its container. As the force required squeezing out the emulgel from its container increases, its extrudability also increases. Greater the extrudability value, the lesser the spreadability of the formulation. Extrudability values of P. guajava emulgels range from 11.13 to $16.10 \mathrm{~g} / \mathrm{cm}^{2}$. All emulgel formulations were found to be satisfactory to be extrudable. P. guajava Linn. emulgels are shown in Table 5.

\section{Antibacterial activity}

The Antigua well-diffusion technique was used to investigate the antimicrobial activity of $P$. guajava emulgels. The medium used was Mueller-Hinton agar. Gram-positive (S. aureus ATCC25923) and
Gram-negative (P. aeruginosa ATCC 27853) microorganisms have been chosen for antimicrobial studies as the main bacteria to cause oral problems [13]. Table 6 displays antibacterial activities against different strains of bacteria such as $S$. aureus and P. aeruginosa in the emulgels formulations of $P$. guajava and comparison with marketed antifungal formulation (Daktarin oral gel). The table affirmed that the antibacterial activity of all formulations was contrary to Gram-negative and Gram-positive bacteria.

\section{DISCUSSION}

The present study was intended development of emulgel formulations of $P$. guajava Linn. leaves extract with clove oil and to check the efficacy of antimicrobial activity $P$. guajava Linn. leaves extracts in comparison to marketed formulation. The solvent extracts of $P$. guajava Linn. leaves were observed to show antimicrobial activity and therefore proved that P. guajava extract might have an effective in vivo antibacterial activity.

\section{CONCLUSION}

The different formulation of $P$. guajava Linn. leaves extract was prepared using of Carbopol 934 polymers with different concentration (F1, F2, F3, F4, and F5). All the formulations were evaluated for physical appearance, $\mathrm{pH}$, homogeneity, spreadability, viscosity, and antimicrobial activity. Formulation $\mathrm{F}_{2}$ was found to be the best formulation and having all acceptable parameters which makes this emulgel formulation of $P$. guajava Linn. leaves extract is good candidate for preventive oral care preparation.

\section{REFERENCES}

1. Khullar R, Saini S, Sethi N, Rana AC. Emulgel: A surrogate approach for topically used hydrophobic drugs. Int J Pharm Biol Sci 2011;1:117-128.

2. Mohamed MI. Topical emulsion gel composition comprising diclofenac sodium. AAPS J 2004;6:26.

3. Baibhav J, Rana AC, Seema S, Vikas S. Emulgel: A comprehensive review on the recent advances in topical drug delivery. Int Res J Pharm 2011;2:66-70.

4. Mohamad MI. Optimization of chlorphenesin emulgel formulation. AAPS J 2004;6:1-5.

5. Panigrahi L, Ghosal SK, Pattnaik S, Maharana L, Barik BB. Effect of permeation enhancers on the release and permeation kinetics of lincomycin hydrochloride gel formulations through mouse skin. Indian J Pharm Sci 2006;68:205-11.

6. Sanjay, Jain BD, Padsalg A, Patel K, Mokale V. Formulation, development, and evaluation of fluconazole gel in various polymer bases. Asian J Pharm 2007;1:63-8.

7. Gupta GD, Gaud RS. Release rate of tenoxicam from acrypol gels. Indian Pharm 2005;1:69-76.

8. Bhanu PV, Shanmugam, Lakshmi PK. Development, and optimization of novel diclofenac emulgel for topical drug delivery. Pharm Glob 2011;9:1-4.

9. Obasi NL, Egbuonu AC, Ukoha PO, Ejikeme PM. Comparative phytochemical and antimicrobial screening of some solvent extracts of Samanea saman pods. Afr J Pure Appl Chem 2010;4:206-12.

10. Magaldi S, Mata-Essayag S, de Capriles CH, Perez C, Colella MT, Olaizola $\mathrm{C}$, et al. Well diffusion for antifungal susceptibility testing. Int J Infect Dis 2004;8:39-45.

11. Wood JH, Catacalos G, Liberman SV. Adaptation of commercial viscometers for special applications in pharmaceutical rheology-II. J Pharm Sci 1963;52:375-8.

12. Chakole CM, Shende MA, Khadatkar SN. Formulation and development of novel combined halobetasol propionate and fusidic acid ointment. Int J Chem Technol Res 2009;1:103-16.

13. Valgas C, De Souza SM, Smânia EF, Smânia A Jr. Screening methods to determine antibacterial activity of natural products. Braz J Microbiol 2007;38:369-80. 